\title{
Losses of temporal modulation sensitivity in retinal degenerations
}

\author{
WILLIAM SEIPLE, VIVIENNE GREENSTEIN, AND RONALD CARR \\ From the Department of Ophthalmology, New York University Medical Center New York, New York, USA \\ 10016
}

SUMmARY Sensitivity losses in patients with retinitis pigmentosa (RP) have been attributed to a decrease in photopigment density, to a reduction in the number of photoreceptors, and also to a change in temporal response properties of the receptors. The sensitivity losses in patients with macular degeneration have also been attributed to a loss of photoreceptors. To test these explanations for sensitivity loss we obtained electrophysiological and psychophysical temporal modulation transfer functions (MTFs) on normal subjects in response to varying stimulus luminances and retinal loci. These stimulus manipulations did not duplicate the changes observed in the temporal MTFs of patients. The temporal response properties of the receptors were tested electrophysiologically by manipulating stimulus presentation interval. The results provided evidence for sensitivity losses in RP patients being due to alterations in the temporal response properties of the receptors.

Retinitis pigmentosa (RP) is a heterogenous group of retinal disorders which is associated with a progressive degeneration of the photoreceptors. Most patients with this disorder present with subjective symptoms indicative of a disorder of the rod system; however, there is ample evidence for cone system involvement as well. ${ }^{1-8}$ Stargardt's disease is an autosomal recessive juvenile macular degeneration involving atrophy of the retinal pigment epithelium (RPE) and associated progressive degeneration of the photoreceptors. Whereas in RP peripheral vision is initially affected, in Stargardt's disease the macular area is primarily affected. ${ }^{910}$

In a previous study we assessed changes in cone system temporal sensitivity in patients with these retinal diseases using a focal electroretinographic technique (FERG). ${ }^{6}$ Both RP and Stargardt's disease patients showed amplitude losses over the range of temporal frequencies tested; however, the pattern of loss differed between the two groups. RP patients with good visual acuities showed FERG amplitude losses at the higher temporal frequencies. In RP patients with decreased visual acuity their was an overall amplitude loss which was most severe at the higher temporal frequencies. In contrast, patients with Stargardt's disease had FERG amplitude losses Correspondence to W Seiple, PhD. at both the low and high temporal frequencies, with responses to the mid-temporal frequencies being relatively spared (see insert to Fig. 3).

Various hypotheses have been proposed to account for the sensitiyity losses of the cone system in these diseases. One explanation for RP attributes a major component of sensitivity loss to a decrease in quantum catching ability of the photoreceptors. This could result from a decrease in photopigment density, a misalignment of photoreceptors, or a shortening of photoreceptors. Histological examination of RP retinas, psychophysical studies of RP patients by colour matching techniques, and foveal densitometric studies all lend support to this explanation. ${ }^{711-13}$

Another explanation for the observed temporal sensitivity losses in RP patients suggests that time constants, or the dynamic adaptation properties, of the cones of the central retina are changed. This deficit would have the greatest effect on sensitivity to higher temporal frequency stimuli. ${ }^{6}{ }^{14}$ is

The changes in sensitivity exhibited by patients with Stargardt's disease have also been explained by a decrease in quantum catching ability of the cone system. Indeed a recent foveal densitometric study of patients with Stargardt's disease reported low two-way densities. ${ }^{16}$ Another explanation for the 
observed changes in Stargardt's disease suggests that, as central cones are lost, temporal sensitivity may be increasingly determined by peripheral retinal elements.

In this study we attempt to test experimentally these mechanisms of sensitivity loss by observing the changes in the focal electroretinogram (FERG) and psychophysically elicited flicker thresholds of normal subjects in response to changes in stimulus luminance and as a function of retinal location. We do not attempt directly to compare psychophysical and electrophysiological findings. Instead, FERG findings are compared with previous electrophysiological results obtained in RP and Stagardt's disease

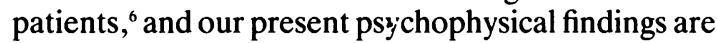
compared with those of Tyler et al.${ }^{14}$ from RP patients and with those of a Stagardt's disease patient reported on by Kayazawa et al. ${ }^{17}$ In a second experiment changes in response time constant were examined by measuring FERG response amplitude changes as a function of stimulus presentation interval. The results in normal subjects were compared with those in patients with RP.

\section{Material and methods}

Stimulus. An array of red LEDs subtending $9^{\circ}$ at a viewing distance of $33 \mathrm{~cm}$ was centred in an illuminated Ganzfeld bowl. The LEDs were driven sinusoidally at a modulation depth of $100 \%$. In each session five temporal frequencies ranging from 10 to $50 \mathrm{~Hz}$ were presented to the subject. The mean luminance of the stimulus array and surround could be adjusted independently (see Seiple et al. ${ }^{6}$ for a complete description of the stimulus apparatus).

$F E R G$ recording. After pupil dilatation (1\% tropicamide) and topical anaesthesia $(0.5 \%$ proparacaine hydrochloride), monocular FERG responses were recorded with gold foil electrodes. ${ }^{18}$ The ipsilateral ear served as the reference and the contralateral ear as ground. The other eye was occluded throughout the test. ${ }^{19}$ The retinal potentials were amplified at a gain of 10000 (Grass P511), and the filters were adjusted so that no signal attenuation occurred at the frequency of stimulation. Responses were signal averaged with artefact rejection (Nicolet $1170, n=128$ ) and plotted on an X-Y plotter for analysis.

Psychophysical testing. Threshold responses were obtained psychophysically with the same stimulus apparatus. The modulation depth of the LED array was slowly increased by means of a hand-held potentiometer until the subject reported detection of flicker. Four ascending trials were averaged for each subject at each stimulus condition.

Normal subjects. Two of the authors (WS and VG) and two students participated in this study. All were acquainted with the purpose of the experiments.

Stimulus manipulations. Experimental stimulus manipulations were chosen to test the hypotheses discussed above.

(1) Decreased quantal catch hypothesis (decrease in the luminance of both the test and surround):

The effects of a decrease in the effective intensity of the stimulus on temporal modulation sensitivity were examined by placing a $0 \cdot 3$ neutral density filter in front of the tested eye. This was done to mimic the effects of a loss of $50 \%$ of the visual pigment. This experimental manipulation equally reduced the test and the surround luminance. We predicted from previous psychophysical work that under the neutral density filter condition there should be decrease in sensitivity at all temporal frequencies, with sensitivity being less affected for the higher frequencies. ${ }^{14} 2021$

(2) Relative contribution of the fovea (decrease in test luminance only):

In Stargardt's disease the central retina is primarily affected. Thus there might be relatively less contribution by the foveal elements and a greater relative contribution of more peripheral elements to the response. Although this is hard to mimic in normal persons, we attempted to do so by reducing the mean luminance of the stimulus while leaving the periphery unchanged. The test luminance was decreased by $0 \cdot 3$ $\log$ unit, and measurements were obtained after adapting to the new level for 5 minutes.

The relative contribution of the foveal versus the peripheral elements was also tested by imaging the stimulus eccentrically. This was done in order to assess whether the temporal sensitivity of the peripheral retina in normal subjects resembled the pattern of temporal sensitivity observed in the diseased retina. The stimulus was centred at $10^{\circ}$ temporal to the fovea by directing fixation to an appropriate target on the Ganzfeld bowl.

(3) Changes in temporal response properties:

Changes in time constant of the diseased retina were examined by a paradigm in which the interval between two flashes was parametrically varied (a twin flash FERG paradigm). From our previous work $^{6}$ we expected that patients with RP would show an increased time constant. This should result in a prolongation of recovery to the first flash and a subsequent decrease in response amplitude to the second flash. This effect should occur at longer interstimulus intervals in the RP patients than in the normals.

In this experiment pairs of square wave pulses (10 ms duration) were presented by the focal ERG apparatus described above. The time interval between pulse pairs (interstimulus interval) was 
FOCAL ERG
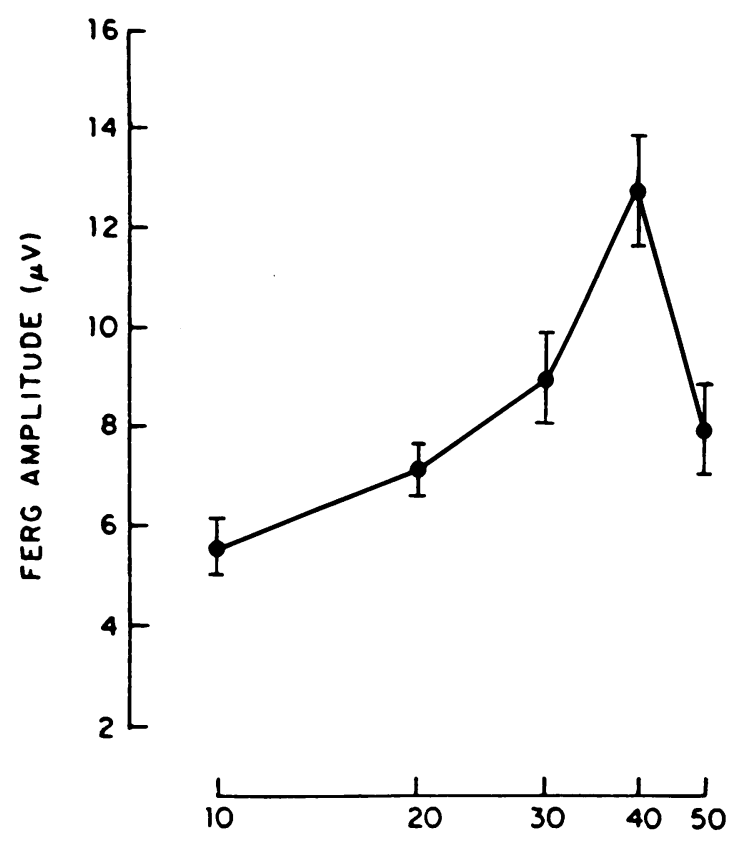

TEMPORAL FREOUENCY $\left(\mathrm{HZ}_{\mathbf{Z}}\right)$

Fig. 1 Mean amplitude $( \pm 1 S D)$ for normal subjects under the 'standard' stimulus condition $\left(50 \mathrm{~cd} / \mathrm{m}^{2}, 100 \%\right.$ modulation, central fixation).

varied from 150 to $40 \mathrm{~ms}$, with the intertrial interval constant at $500 \mathrm{~ms}$. The ERG response to each stimulus condition was averaged $(n=128)$. Six normal subjects and five RP patients (two females and three males ranging in age from 45 to 66) were tested in this experiment. The patients' visual acuities were 20/20 $(n=2), 20 / 30(n=2)$, and 20/70 $(n=1)$. All patients had central fields subtending at least $10^{\circ}$ measured with a III-4 white test light in a Goldmann perimeter. These patients were chosen to span a large range of macular involvement.

\section{Results}

The FERG responses to the various experimental manipulations were compared with FERG responses obtained under the 'standard' condition (mean luminance $=50 \mathrm{c} / \mathrm{m}^{2}$, modulation depth $=100 \%$, and the array centrally fixated). Percentage amplitude loss was calculated by the following formula ((standard response amplitude minus experimental response amplitude)/standard response amplitude $\times$ 100). For the psychophysically determined data percentage sensitivity loss was calculated in the same
PSYCHOPHYSICS

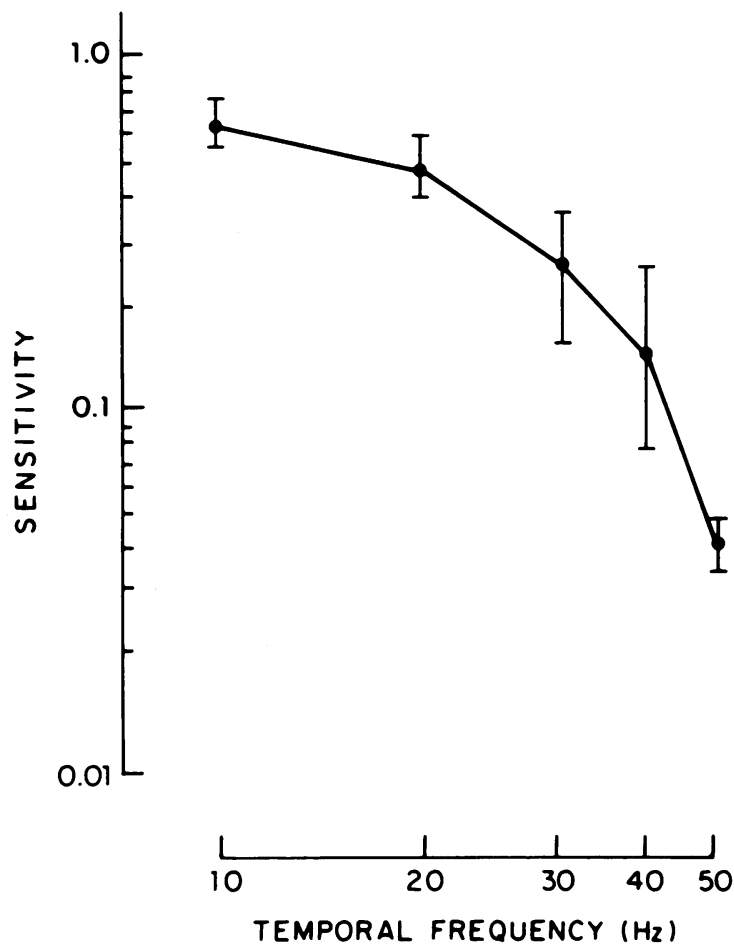

Fig. 2 Mean sensitivity ( $\pm 1 S D)$ measured psychophysically for the normal subjects under the 'standard' condition (50 cd/ $\mathrm{m}^{2}, 100 \%$ modulation, central fixation).

manner by comparing the flicker thresholds obtained under various experimental conditions with those obtained under the 'standard' condition.

Responses to the 'standard' condition. Mean FERG amplitude as a function of temporal frequency is presented in Fig. 1. For normal subjects there was a maximum response at $40 \mathrm{~Hz}$, with a sharp high frequency and a shallower low frequency amplitude fall-off. These data are similar to the FERG data previously presented from this laboratory. ${ }^{6}$

Temporal frequency sensitivity obtained psychophysically is presented in Fig. 2. Peak sensitivity occurred at $10 \mathrm{~Hz}$, with a loss of sensitivity at higher temporal frequencies. These data are similar to those previously presented. ${ }^{14} 2021$

Decreased quantal catch hypothesis. Fig. 3 presents the mean percentage FERG amplitude losses for four normal subjects in response to a $\mathbf{0 \cdot 3}$ neutral density filter placed in front of the tested eye. The presence of the filter resulted in a response amplitude increase at the low frequencies and an amplitude decrease at the intermediate temporal frequencies. However, at $50 \mathrm{~Hz}$ the percentage loss was less than that observed 
FOCAL ERG
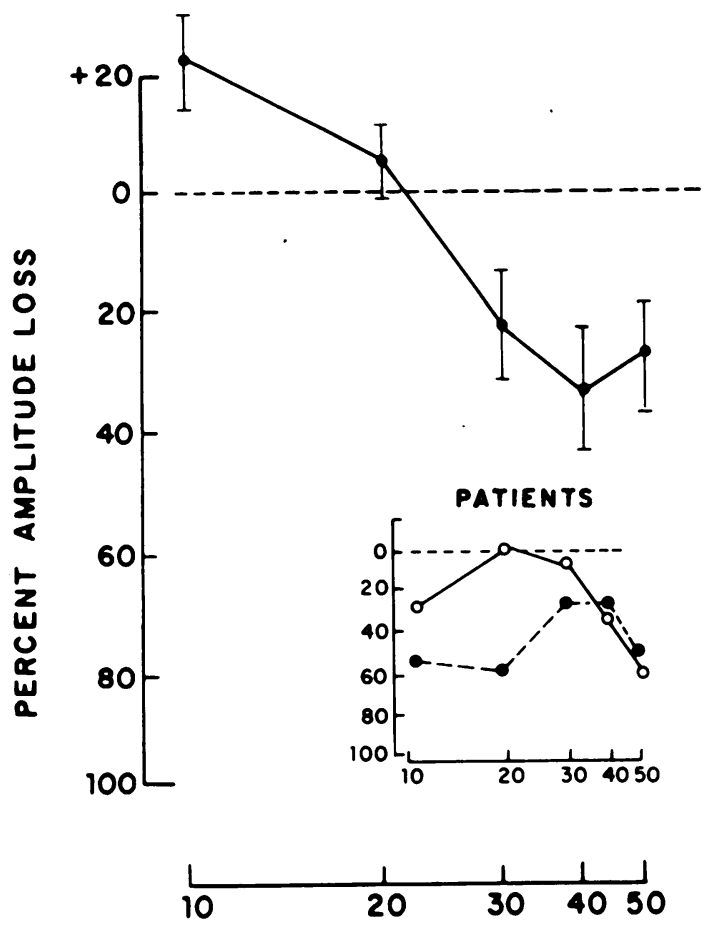

TEMPORAL FREQUENCY (Hz)

Fig. 3 Percentage FERG amplitude loss under the 0.3 neutral density filter condition. Amplitude to the experimental condition is compared with the amplitude obtained under the 'standard' condition. If the two conditions produced the same amplitude responses, the plot would be a horizontal line at $0 \%$ amplitude loss. A positive percentage amplitude loss indicates that the response under the experimental condition was greater than that obtained under the standard condition, and a negative percentage amplitude loss denotes that the response amplitude was less under the experimental condition. The insert shows the corresponding FERG data for RP patients (open circles) and Stargardt's disease patients (filled circles; from Seiple et al.').

at $40 \mathrm{~Hz}$. Psychophysical results for this stimulus condition are shown in Fig. 4. Sensitivity to $10 \mathrm{~Hz}$ was relatively unaffected, and there was a greater sensitivity loss at the middle temporal frequencies. Once again at $50 \mathrm{~Hz}$ the relative sensitivity loss was less that observed at the intermediate frequencies. The corresponding electrophysiological and psychophysical results for RP and Stargardt's disease patients are presented in the inserts in the figures for comparison. The inserts are from Seiple et al. ${ }^{6}$ and represent FERG results obtained from 10 RP patients with good visual acuity (20/20) and from three patients with Stargardt's disease who had a visual acuity of $20 / 40$ or better. We chose these

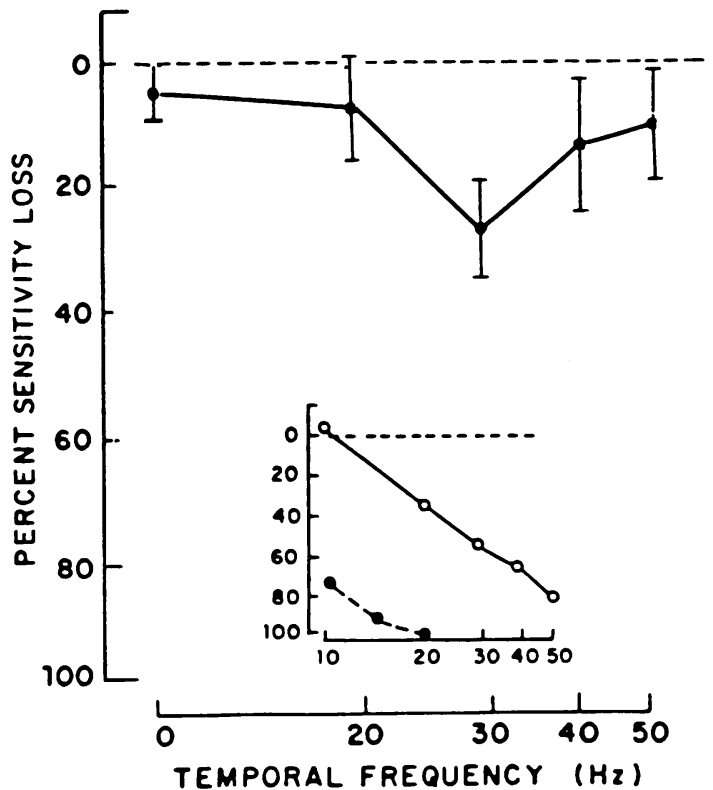

Fig. 4 Percentage sensitivity loss measured psychophysically under the 0.3 neutral density filter condition. Sensitivity to the experimental condition is compared with the sensitivity measured under the 'standard' condition. If the two conditions produced the same threshold responses, the plot would be a horizontal line at $0 \%$ amplitude loss. The insert presents corresponding psychophysically obtained data for RP patients (open circles, from the data presented by Tyler et $\left.\mathrm{al}^{.14}{ }^{14}\right)$ and for one Stargardt's disease patient (filled circles; from Kayazawa et al. ${ }^{17}$ ).

patients for comparison because they showed the changes in temporal processing seen at the early stages of the diseases. The insert on Fig. 4, presenting psychophysical data, is the mean of 11 RP simplex patients of Tyler et al. ${ }^{14}$ These authors did not distinguish individual patients on their graphical presentation, and therefore the average presented in our insert represents RP patients who had visual acuities ranging from $20 / 20$ to $20 / 80$. The high frequency specific pattern of loss, however, is representative of their findings. The insert data for Stargardt's disease have been derived from a single patient $(\mathrm{VA}=20 / 100)$ presented by Kayazawa et al. ${ }^{17}$ To our knowledge this is the only Stargardt's disease patient in the literature who has been studied by psychophysical techniques.

Relative contribution of the fovea. Fig. 5 presents the FERG results of the reducing stimulus mean luminance (to $25 \mathrm{~cd} / \mathrm{m}^{2}$ ). The largest response amplitude losses occurred for the mid temporal 
FOCAL ERG

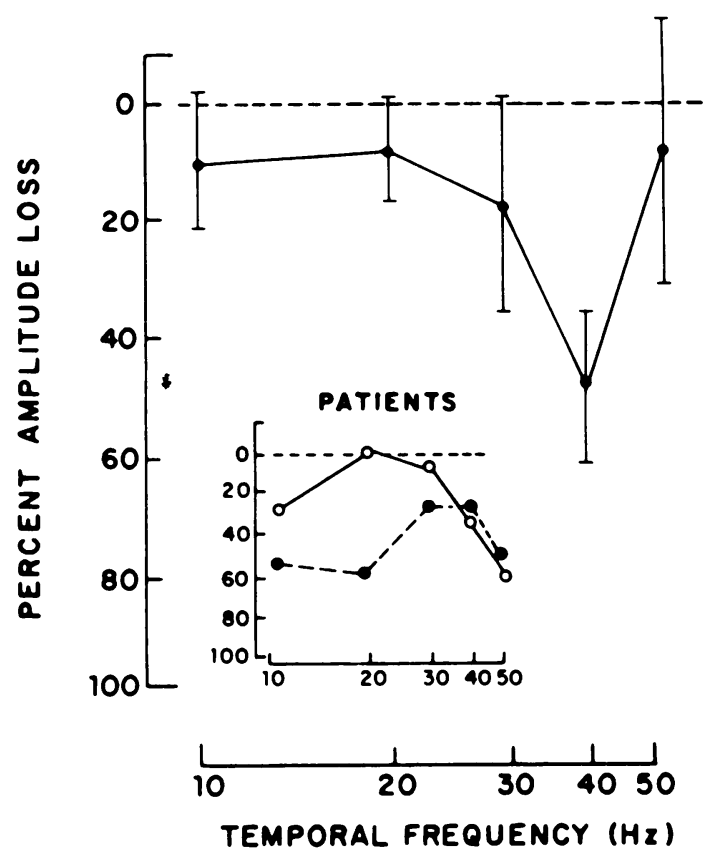

Fig. 5 Percentage FERG amplitude loss obtained when the stimulus luminance was reduced by half $\left(25 \mathrm{~cd} / \mathrm{m}^{2}\right)$. FERG amplitudes obtained in response to this experimental condition are compared with those obtained under the 'standard' condition. The insert shows the corresponding FERG data for RP patients (open circles) and Stargardt's disease patients (filled circles; from Seiple et al. ${ }^{6}$ ).

frequency $(40 \mathrm{~Hz})$, with a relative sparing of both the low and high frequency amplitudes. Fig. 6 shows a similar trend for the psychophysical data. There is no change in sensitivity at the low temporal frequencies $(10$ and $20 \mathrm{~Hz})$, and decrease in sensitivity was observed at 30 and $40 \mathrm{~Hz}$. However, at $50 \mathrm{~Hz}$ the percentage loss of sensitivity was less than that observed for $40 \mathrm{~Hz}$.

The results of stimulating more peripheral retina are presented in Figs. 7 and 8. Electrophysiologically there was an overall decrease in amplitude at all temporal frequencies $(10$ through $50 \mathrm{~Hz})$. The decrease was minimal for the low temporal frequencies and highest for the mid temporal frequencies. As in the other stimulus conditions, the highest temporal frequency $(50 \mathrm{~Hz})$ responses showed relatively less percentage amplitude loss than the mid temporal frequencies ( 30 and $40 \mathrm{~Hz}$ ). Psychophysically there was an increase in sensitivity at the higher temporal frequencies $(>30 \mathrm{~Hz})$ as compared with the 'standard' central fixations condition (Fig. 8).

Changes in temporal response properties (twin flash

\section{PSYCHOPHYSICS}

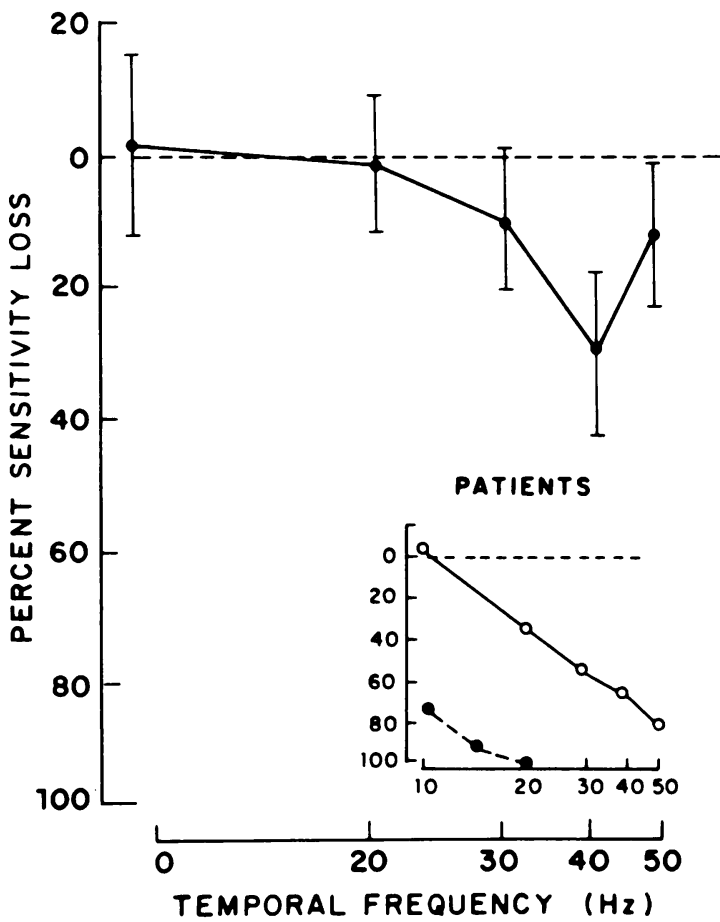

Fig. 6 Percentage sensitivity loss measured psychophysically under the $25 \mathrm{~cd} / \mathrm{m}^{2}$ stimulus luminance condition. Sensitivity to the experimental condition is compared with the sensitivity measured under the 'standard' condition. The insert shows corresponding psychophysically obtained data for RP patients (open circles, from the data presented by Tyler et al. ${ }^{\text {'4) }}$ and for one Stargardt's disease patient (filled circles; from Kayazawa et al. ${ }^{17}$ ).

paradigm). In the normal subjects the amplitude of the response to the second flash gradually changed as a function of interstimulus interval. The complete curve is presented for only one normal subject (solid line in Fig. 9). Six normal subjects showed their lowest amplitude ERG responses to the second flash within the interstimulus interval ranging between 40 and $60 \mathrm{~ms}$ (filled circles, mean interval $=49 \cdot 75, \mathrm{SD}$ 3.77). The complete data for one RP patient are shown by the dotted curve. Five RP patients (open circles, Fig. 9) with relatively good visual acuity showed their lowest amplitude ERG response to the second flash in the interval between 60 and $80 \mathrm{~ms}$ (mean interval $=71 \cdot 6$, SD 6.06). The difference between the means of the two groups was statistically significant (Student's $t=4 \cdot 99, \mathrm{p}<0 \cdot 002, \mathrm{df}=9$ ).

\section{Discussion}

We have previously presented electrophysiological 


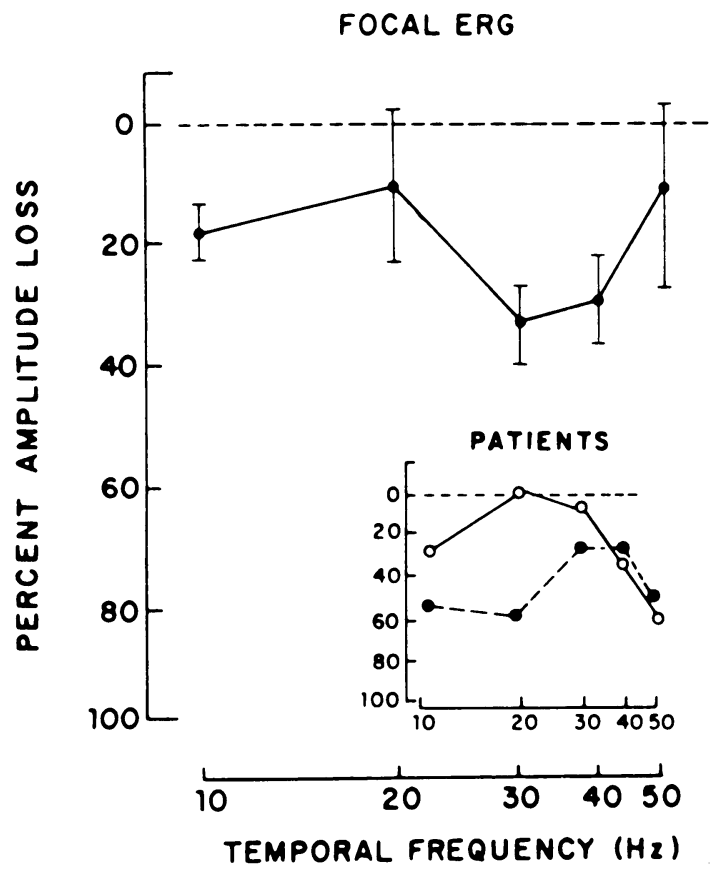

Fig. 7 Percentage FERG amplitude loss under the $10^{\prime \prime}$ fixation condition. Amplitude to the experimental condition is compared with the amplitude obtained under the 'standard' condition. The insert shows the corresponding data for $R P$ patients (open circles) and Stargardt's disease patients (filled circles; from Seiple et al.").

evidence that there is a progressive loss in FERG amplitude at the higher temporal frequencies in RP patients with good visual acuity. ${ }^{6}$ From our current electrophysiological findings such a loss cannot be attributed to a decrease in the effective luminance of the stimulus - that is, decrease in quantum catching ability. The FERG responses obtained by experimentally manipulating the stimulus intensity showed relatively less amplitude loss at the highest temporal frequency compared with the mid temporal frequencies. Similarly, Massof et al. ${ }^{15}$ in a later study, showed that the $b$ wave latency changes at high temporal frequencies observed in a group of RP patients cannot be mimicked by decreasing retinal adaptation by changing the stimulus intensity.

The findings of a progressive FERG amplitude loss at high temporal frequencies in RP patients has led us to suggest that such losses may be due to a change in temporal properties of the receptors. ${ }^{6}$ In fact our twin flash results (Fig. 9) provide evidence for an increased time constant. These changes appear to have their origin in the outer retina, since FERG responses most probably reflect receptor activity. ${ }^{22}$ Support for this contention is derived from a number of lines of evidence. Studies on the intact macaque

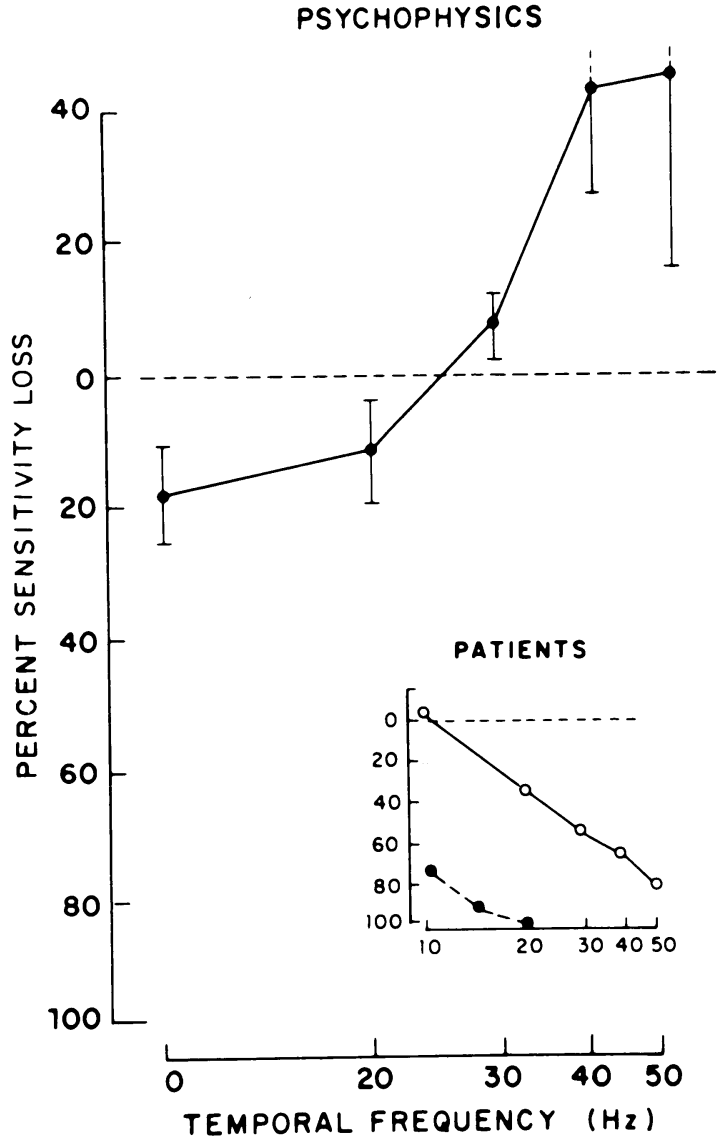

Fig. 8 Percentage sensitivity loss measured psychophysically under the $10^{\prime \prime}$ fixation condition. Sensitivity to the experimental condition is compared with the sensitivity measured under the 'standard' condition. The insert shows corresponding psychophysically obtained data for $R P$ patients (open circles, from the data presented by Tyler et al. $\left.{ }^{14}\right)$ and for one Stargardt's disease patient (filled circles; from Kayazawa et al. ${ }^{17}$ ).

monkey indicate that responses to high temporal frequency sinusoidal stimulation are derived from the outer retina. ${ }^{23}$ Local ERG responses recorded in the monkey showed no intrusion of the ERG b wave, and both high and low frequency components of peripheral and central retinal stimulation have been shown to derive from the receptor activity. ${ }^{24}$ In addition, Donovan and Baron ${ }^{25}$ have shown that the corneal positive component of the monkey ERG recorded in response to a sinusoidally flickering stimulus is in fact a distal representation of the foveal local electroretinogram (LERG). Further support for the outer retinal origin of the fast flicker responses is the failure of glial cells to follow high flicker rates. ${ }^{26}$

It is interesting to note, however, that the a wave 


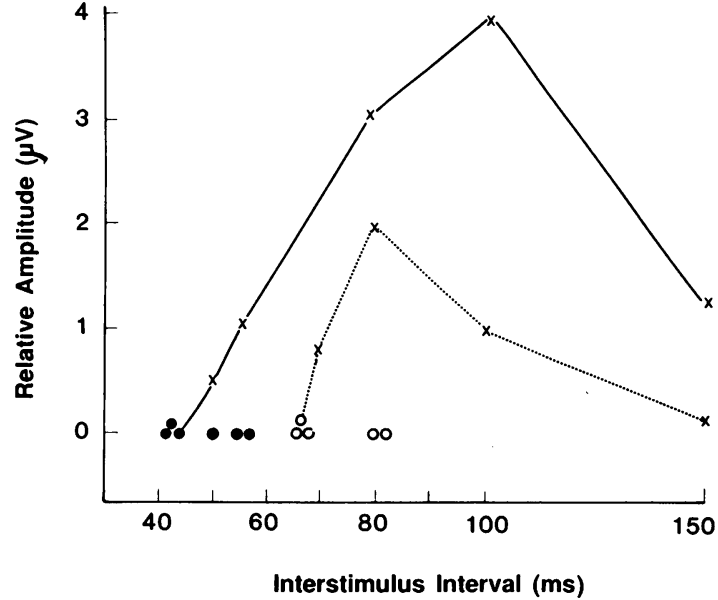

Fig. 9 Relative ERG amplitudes to the second of a pair of flashes for six normal subjects (filled circles) and five RP patients (open circles) plotted as a function of interstimulus interval. The response amplitudes for all data have been normalised by setting each subject's lowest amplitude to $0 \mu V$. The entire curves for only one normal (solid line) and one RP patient (dotted line) are presented for comparison. For the other subjects only the corresponding interstimulus interval at which the response amplitude was lowest is indicated.

latencies (which reflect outer retinal processing) were normal in the RP patients studied by Massof et al. ${ }^{15}$ This finding is not inconsistent with the proposed changes in time constant mentioned above. A delay in the time to recover following a flash may not necessarily affect time to respond (that is, latency). Instead it could primarily affect the amplitude of subsequent responses if these responses occurred within this prolonged refractory period. Indeed we have shown that the inhibitory effects of an initial flash are prolonged in RP patients (Fig. 9). FERG amplitudes have also been found to be reduced at high temporal frequencies (where stimuli follow at short intervals). However, the phase (latency) of the FERG for this group of RP patients was normal at all temporal frequencies regardless of visual acuity loss. ${ }^{6}$

Our electrophysiological findings in patients with Stargardt's disease are also not consistent with an explanation based on a decrease in quantum catching ability. These patients showed losses of response amplitude at both low and high temporal frequencies, with relative sparing of the mid temporal frequency response amplitude. ${ }^{6}$ Again these changes in temporal responsiveness do not resemble the changes observed when the effective luminance of the stimulus was reduced. In addition, an explanation for these patients' temporal losses based on a relatively greater contribution of peripheral retina to the response must be rejected. The present data show that peripheral retinal stimulation results in the greatest decrease in FERG amplitude at mid temporal frequencies.

The pattern of psychophysical sensitivity loss in normal persons in response to decreased stimulus luminance also differs from the pattern of psychophysical loss found in patients. Instead of a progressive loss at high temporal frequencies in both RP and Stargardt's disease patients we found that the sensitivity loss at $50 \mathrm{~Hz}$ was relatively less than that at 30 or $40 \mathrm{~Hz}$. These findings are in agreement with psychophysical data obtained by deLange ${ }^{20}$ and Kelly. ${ }^{21}$ Tyler et al. ${ }^{14}$ have suggested that the differences in the patterns of psychophysical flicker sensitivity observed in their RP patients are due to changes in the responsiveness of the retinal elements. Using a probe flash technique, Greenstein and $\mathrm{Hood}^{27}$ have also suggested that cone system sensitivity changes in their RP patients are due to a decreased responsiveness of retinal elements rather than a decrease in quantal catch.

Similarly, the pattern of psychophysical flicker sensitivity changes we observed by stimulating more peripheral retina does not resemble the pattern observed in Stargardt's disease.$^{17}$ Instead of a decrease in sensitivity at the high temporal frequencies, normal subjects show an enhancement of flicker sensitivity in the peripheral retina. This increased high frequency flicker sensitivity of the peripheral retina is consistent with previous psychophysical reports. ${ }^{28} 29$

\section{CONCLUSION}

Neither the psychophysical nor the electrophysiological data support an explanation of cone system temporal sensitivity losses observed in patients with RP or Stargardt's disease based solely on a decrease in the quantum catching ability of photoreceptors. We propose that the high frequency sensitivity loss of these patients may be based on changes in time constants. Our twin flash data suggest that this may indeed be the case.

This work was supported by a grant from the RP Foundation and Allied Diseases to the NYU Retina Clinic and NEI grant EY02115. We thank Don Hood and Karen Holopigian for their helpful comments on an earlier version of this manuscript.

\section{References}

1 Greenstein VC, Hood DC, Siegel IM, Carr RE. Retinitis pigmentosa: a psychophysical test of explanations for early foveal sensitivity loss. Invest Ophthalmol Vis Sci 1984; 25: 11820.

2 Massof RW, Finkelstein D. Rod sensitivity relative to cone sensitivity in retinitis pigmentosa. Invest Ophthalmol Vis Sci 1983; 18: 263-72. 
3 Sandberg MA, Jacobson SG, Berson EL. Foveal electroretinograms in retinitis pigmentosa and juvenile macular degeneration. Am J Ophthalmol 1979; 88: 702-7.

4 Sandberg MA, Berson EL. Visual acuity and cone spatial density in retinitis pigmentosa. Invest Ophthalmol Vis Sci 1983; 27: 1511-3.

5 Seiple WH, Siegel IM, Carr RE, Mayron C. Objective assessment of temporal modulation transfer functions using the focal ERG. Am J Optom Physiol Opt 1986; 63: 1-6.

6 Seiple WH, Siegel IM, Carr RE, Mayron C. Evaluating macular function using the focal ERG. Invest Ophthalmol Vis Sci 1986; 27: 1123-9.

7 van Meel GJ, van Norren D. Foveal densitometry in retinitis pigmentosa. Invest Ophthalmol Vis Sci 1983; 24: 112330 .

8 Young RSL, Fishman GA. Sensitivity loss in a long wavelength sensitive mechanism of patients with retinitis pigmentosa. Vision Res 1982; 22: 163-72.

9 Krill AE. Hereditary retinal and choroidal diseases. New York: Harper and Row, 1977; 2: 1371p.

10 Noble K, Carr R. Stargardt's disease and fundus flavimaculatus. Arch Ophthalmol 1979; 97: 1281-5.

11 Alexander KR, Huntman LP, Fishman GA. Dark-adapted foveal thresholds and visual acuity in retinitis pigmentosa. Arch Ophthalmol 1986; 104: 390-4.

12 Kilbride P, Fishman M, Fishman G, Huntman LP. Foveal cone pigment density difference and reflectance in retinitis pigmentosa. Arch Ophthalmol 1986; 104: 220-4.

13 Kolb H, Gouras P. Electron microscope observations of human retinitis pigmentosa, dominantly inherited. Invest Ophthalmol Vis Sci 1974; 13: 487-98.

14 Tyler CN, Ernst W, Lyness AL. Photopic flicker sensitivity losses in simplex and multiplex retinitis pigmentosa. Invest Ophthalmol Vis Sci 1984; 25: 1035-42.

15 Massof RW, Johnson MA, Sunness JS, Perry C, Finklestein D. Flicker electroretinogram in tetinitis pigmentosa. Doc Ophthalmol 1986; 62: 231-45.

16 van Meel GJ, van Norren D. Foveal densitometry as a diagnostic technique in Stargardt's disease. Am J Ophthalmol 1986; 102: 353-62.
17 Kayazawa F, Sonoda K, Nishimura K, Nakamura T, Yamaoto T, Itoi M. Clinical application of temporal modulation transfer function. Jpn J Ophthalmol 1984; 28: 9-19.

18 Arden GB, Carter RM, Hogg C, Siegel IM, Margolis S. A gold foil electrode: extending the horizons for clinical electroretinography. Invest Ophthalmol Vis Sci 1979; 18: 421-6.

19 Seiple WH, Siegel IM. Recording the pattern electroretinogram: a cautionary note. Invest Ophthalmol Vis Sci 1982; 24: 796-8.

20 deLange $\mathrm{H}$. Relationship between critical flicker frequency and a set of low frequency characteristics of the eye. J Opt Soc Am $1954 ; 44: 380-9$.

21 Kelly DH. Visual response to time-dependent stimuli. 1. Amplitude sensitivity measurements. J Opt Soc Am 1961; 51: $422-9$.

22 Abraham FA, Alpern M, Kirk DB. Electroretinograms evoked by sinusoidal excitation of human cones. J Physiol (Lond) 1985 ; 363: 135-50.

23 Baron WS, Boyton RM. The primate foveal local electroretinogram: an indicator of photoreceptor activity. Vision Res 1974; 14: 495-501.

24 Baron WS, Boyton RM. Responses of primate cones to sinusoidally flickering homochromatic stimuli. J Physiol (Lond) 1975; 246: 311-31.

25 Donovan WJ, Baron WS. Identification of the R-G cone difference signal in the corneal electroretinogram of the primate. J Opt Soc Am 1982; 72: 1014-20.

26 Miller RF, Dowling JE. Intracellular responses of the Müller (glial) cells of the mudpuppy retina: Their relation to b-wave of the electroretinogram. J Neurophysiol 1970; 33: 232-341.

27 Greenstein VG, Hood DC. Test of the decreased responsiveness hypothesis in retinitis pigmentosa. Am J Optom Physiol Opt 1986; 63: $22-7$.

28 Tyler CW. Analysis of visual modulation sensitivity: II. Peripheral retina and the role of photoreceptor dimensions. J Opt Soc Am 1982; 2: 393-8.

29 Seiple WH, Greenstein VG, Holopigian K, Carr RE. Changes in the focal electroretinogram with retinal eccentricity. Doc Ophthalmol in press.

Accepted for publication 6 October 1988. 